05,11

\title{
Особенности определения параметров ориентационных магнитных фазовых переходов в гематите магнитоакустическими методами
}

\author{
(C) С.А. Мигачев ${ }^{1}$, М.И. Куркин ${ }^{2}$ \\ ${ }^{1}$ Казанский фризико-технический институт им. Е.К. Завойского ФИЦ Казанский научный центр РАН, \\ Казань, Россия \\ ${ }^{2}$ Институт фоизики металлов УрО РАН, \\ Екатеринбург, Россия \\ E-mail: smigach.kfti.knc.ru
}

Поступила в Редакцию 7 сентября 2020 г.

В окончательной редакции 7 сентября 2020 г.

Принята к печати 8 сентября 2020 г.

Представлены результаты измерения скорости магнитоупругих волн в гематите, соответствующих их квазифононной ветви. Исследована зависимость данной скорости $(V)$ от магнитного поля $(H)$ при постоянном одноосном давлении $P,\left(V_{P}(H)\right)$ и давления $P$ при постоянном $H,\left(V_{H}(P)\right)$. В соответствии с существующей теорией магнитоакустических явлений зависимости $V_{P}(H)$ и $V_{H}(P)$ должны иметь минимумы в точках ориентационных фазовых переходов. Такие минимумы обнаружены, но их координаты на плоскости $(P, H)$ оказались несовпадающими. Обсуждаются причины несовпадений и требования к точности измерений, обеспечивающие возможности их использования для определения параметров этих переходов.

Ключевые слова: антиферромагнетик, магнитоупругость, ориентационные фазовые переходы, объемная акустическая волна.

DOI: 10.21883/FTT.2021.02.50469.188

\section{1. Введение}

Обменное взаимодействие обычно значительно превосходит все остальные взаимодействия с участием спиновых моментов электронов [1]. Однако оно определяет только их взаимную ориентацию, от которой зависит тип спинового упорядочения (ферро-, ферри-, антиферрои др.) и температура перехода - Кюри $T_{\mathrm{C}}$ или Нееля $T_{\mathrm{N}}$ в парамагнитное состояние. Все остальные характеристики этих веществ, обеспечивающие, в том числе и возможности их технических применений, определяются более слабыми взаимодействиями с кристаллическими полями ионов. К ним относятся константы магнитной анизотропии, магнитострикции, магнитоупругости и др. В частности, магнитоупругость, ответственная за взаимодействие колебаний спинов (магнонов) и ионов решетки (фононов) определяет целое направление в магнетизме, получившее название магнитоакустики. Для технических приложений магнитоакустики важна возможность влияния магнитного поля на параметры звуковых волн. Наиболее сильным это влияние оказывается вблизи ориентационных фазовых переходов, связанных с перестройкой магнитной структуры среды. Вблизи таких переходов имеет место взаимная компенсация основных взаимодействий, ответственных за эту структуру, благодаря чему даже слабое магнитоупругое взаимодействие становится определяющим $[1,2]$.

Теория магнитоакустических явлений вблизи ориентационных фазовых переходов подробно изложена в обзоре Турова и Шаврова [3]. Согласно этой теории скорости звука квазифононной ветви (квазизвука) от магнитного поля $H$ при постоянном давлении $P\left(V_{P}(H)\right)$ и от $P$ при постоянных значениях $H\left(V_{H}(P)\right)$ должны совпадать в точках ориентационных фазовых переходов. При этом в идеальных кристаллах скорости $V_{P}(H)$ и $V_{H}(P)$ являются непрерывными функциями $H$ и $P$ соответственно, достигая минимумов в точках перехода. Однако их производные соответственно по $H$ и $P$ испытывают в этих точках скачки. Наличие таких скачков позволяет фиксировать положения точек перехода на плоскости $(P, H)$ с погрешностью измерения величин $H$ и $P$. В реальных веществах структурные неоднородности и неоднородности деформации, обусловленные давлением $P$, сильно уширяют минимумы $V_{P}(H)$ и $V_{H}(P)$, что создает проблемы двух типов. Во-первых, увеличивается погрешность в определении положения этих минимумов. Она зависит не только от погрешности измерения $V_{P}(H), V_{H}(P), H$ и $P$, но и от ширины минимумов. Во-вторых, погрешность, вычисленная с учетом такого уширения, может достигать значений, которые не позволяют использовать данные измерений $V_{P}(H)$ и $V_{H}(P)$ для определения параметров исследуемого вещества. В настоящей работе обсуждаются условия получения магнитоакустических данных для гематита, обеспечивающих определение магнитоупругого параметра с относительной точностью, не превышающей 0.2 .

\section{2. Образцы, методика измерений и обработка результатов}

Схема проводимых экспериментов, в основном, соответствовала данным работы [4] за исключением некото- 


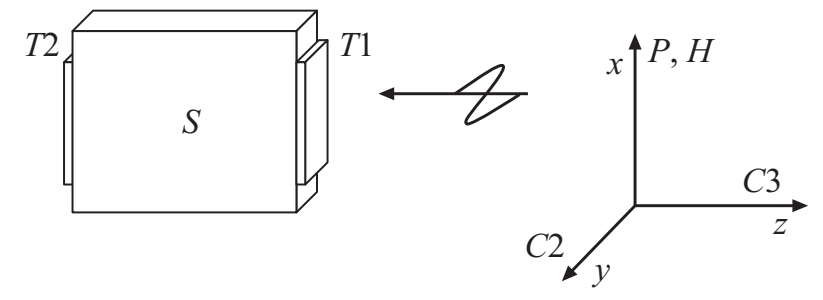

Рис. 1. Схема эксперимента. (S - образец; $T 1, T 2-$ пьезопреобразователи; $P, H-$ сжатие и магнитное поле, направленные вдоль соответствуюших координатных осей; $C 2, C 3$ - кристаллографические оси симметрии гематита.

рых существенных деталей. Все измерения проводились при комнатных температурах на образце гематита $(S)$, которому была придана форма параллелепипеда размерами по осям $x=3.61, y=1.9, z=6.53 \mathrm{~mm}$, как показано на рис. 1. Все грани исследуемого образца гематита были обработаны по оптическим стандартам и были сориентированы относительно кристаллографических осей на рентгеновском дифрактометре с точностью до одного градуса. Выбранная ось симметрии второго порядка $C 2$ была направлена вдоль оси $y$, сжатие образца производилось в базисной плоскости вдоль оси $x$, постоянное внешнее магнитное поле Н прикладывалось также вдоль этой оси.

Для акустических измерений использовалась схема на прохождение. Сдвиговые акустические колебания на частотах $\sim 5.7 \mathrm{MHz}$, возбуждались пластинчатыми пьезопреобразователями $\mathrm{LiNbO}_{3} x$-среза ( $T 1$ и $T 2$ ), присоединенными на твердой склейке, направление распространения звука $(\mathbf{k})$ - вдоль оси симметрии третьего порядка $C 3$ (ось $z$, размер $6.53 \mathrm{~mm}$ ), плоскость поляризации акустических колебаний была перпендикулярна базисной плоскости гематита и содержала в себе выбранную ось $C 2$. Таким образом, возбуждалась взаимодействующая с осцилляциями вектора антиферромагнетизма $\mathbf{L}$ магнитозависимая $\xi$-мода колебаний, скорость которой $V_{\xi}$ (все в обозначениях работ $\left.[5,6]\right)$. Регистрация изменения скорости осуществлялась с использованием двухлучевого цифрового осциллографа TDS 2012 С по сдвигу фазы $\Delta t$ прошедших через образец акустических колебаний (на T2) относительно фазы входного сигнала (на $T 1)$.

Следует отметить некоторые важные детали. Удовлетворительные результаты были получены при использовании для присоединения пьезопреобразователей на твердой склейке минимальной толщины цианоакрилатного клея Permabond 910. Особое внмание было уделено выбору ориентации пьезопреобразователей (относительно кристаллографических осей кристалла), определяющей направление плоскости поляризации акустической волны, генерируемой пьезопреобразователями. Ошибка на углы, даже в несколько градусов, приводит к одновременному возбуждению в образце, наряду с взаимодействующей с осцилляциями вектора антиферромагнетиз- ма $\xi$-модой, еще и невзаимодействующей акустической $\eta$-моды [5-7]. В таком случае, при значительном уменьшении амплитуды $\xi$-моды в условиях ориентационного фазового перехода, присутствие акустической $\eta$-моды, также детектируемой на приемном пьезопреобразователе $T 2$, может вносить погрешность в результаты экспериментов.

Абсолютное значение скорости распространения квазизвука определялось по частотам размерных акустических резонансов образца при приложении сильного магнитного поля $(\mathbf{H}>5 \mathrm{kOe})$ на частотах $\sim 20 \mathrm{MHz}$, когда затухание квазизвука сильно ослабевало [4,8] и размерные резонансы были хорошо выражены с добротностью $Q \sim 100$.

В экспериментах для приложения направленного сжатия $(\mathbf{P})$ до величин $\sim 180 \mathrm{MPa}$, использовалось устройство, сходное с примененным в работе [9], т.е. усилие сжатия прикладывалось к образцу по схеме: „рычаг-шток-образец“. В большинстве устройств для одноосного сжатия используются образцы, имеющие в поперечном (относительно направления сжатия) сечении круг или квадрат, что диктуется условиями сохранения однородности распределения прикладываемого сжатия. В нашем же случае для повышения точности измерения скорости звука возникла необходимость использования образцов с прямоугольным сечением более вытянутых в направлении распространения звука $(6.53 \times 1.9 \mathrm{~mm})$. Это, в свою очередь, приводит к необходимости обеспечить более равномерное сжатие по всей сжимаемой поверхности. Поэтому при конструировании и изготовлении ячейки для проведения экспериментов особое внимание уделялось плоскостности и параллельности перемещения рабочих поверхностей штоков и, тем самым, обеспечения равномерности сжатия. Для этого поверхности движущегося штока и неподвижно закрепленного после тщательной юстировки основания, на котором располагался образец, выполненные из титанового сплава ВТ7, также были обработаны по оптическим стандартам. Для предотвращения разрушения образца, между его сжимаемыми поверхностями и поверхностями штока и основания вкладывалась специально изготовленная фольга толщиной $\sim 100 \mu \mathrm{m}$ из оловянно-свинцового припоя ПОС 40.

На рис. 2 и 3 приведены полученные данные по изменению скорости квазизвука в результате совместного воздействия на образец внешнего магнитного поля $H$ и одноосного давления $P$. Процедура экспериментов была стандартной. Для зависимости $V_{P}(H)$ (рис. 2): 1 прикладывалось давление $P ; 2$ - последовательно при каждом фиксированном значении магнитного поля $H$, производилось измерение сдвига фазы; 3 - поле $H$ уменьшалось до минимального значения ( 100 Ое); 4 - ступенчато увеличивалось давление $P$ и процедура повторялась. Для регистрации зависимости $V_{H}(P)$ (рис. 3) процедура была аналогичной, здесь давление $P$ и поле $H$ по порядку действия менялись местами. 


\section{3. Обсуждение результатов}

Зависимости скорости квазизвука $V_{\xi}(P, H)$ от $P$ и $H$ представлены на рис. 2 и рис. 3 в двух вариантах. $1-$ от $H$ при $P=$ const на рис. $2\left(V_{P}(H)\right) ; 2-$ от $P$ при $H=$ const на рис. $\left(V_{H}(P)\right)$. Теоретические кривые $V_{P}(H)$ и $V_{H}(P)[3]$ претерпевают изломы в точках ориентационных фазовых переходов с изменением знака производных $\partial V_{P}(H) / \partial H$ и $\partial V_{H}(P) / \partial P$. Уширение этих особенностей, обусловленное неоднородностями образца, превращает их в минимумы, ширина которых растет с увеличением $H$ и $P$. При этом возрастает погрешность в определении положения этих минимумов, обусловленная погрешностью измерений $V_{P}(H)$ и $V_{H}(P)$.

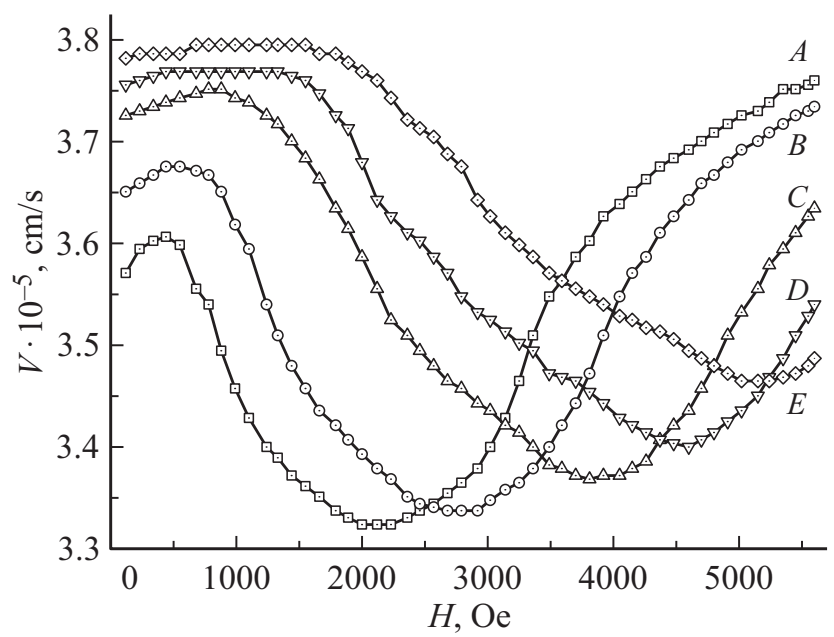

Рис. 2. Зависимость скорости квазизвука $V_{P}(H)$ от магнитного поля $(H)$ для ряда величин приложенных к образцу сжатий $(P)$. $(A-32.56 \mathrm{MPa} ; B-48.23 \mathrm{MPa} ; C-72.04 \mathrm{MPa} ; D-$ $88.58 \mathrm{MPa} ; E-106.53 \mathrm{MPa})$.

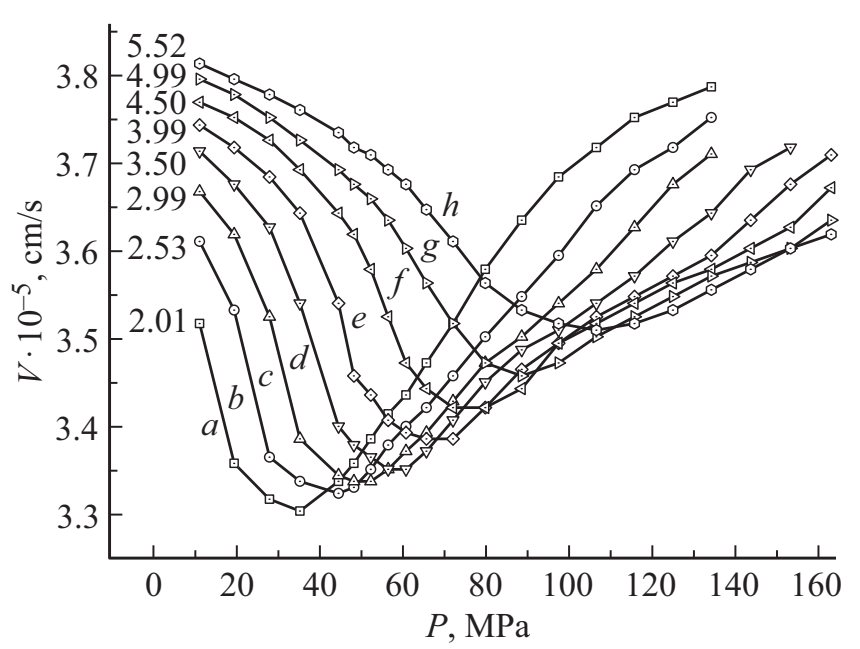

Рис. 3. Зависимость скорости квазизвука $V_{H}(P)$ от приложенных к образцу сжатий $(P)$ для ряда значений приложенных магнитных полей. $(H)$. $(a-2.01 \mathrm{kOe} ; b-2.53 \mathrm{kOe}$; $c-2.99 \mathrm{kOe} ; d-3.5 \mathrm{kOe} V$; $e-3.99 \mathrm{kOe} ; f-4.5 \mathrm{kOe}$; $g-4.99 \mathrm{kOe} ; h-5.52 \mathrm{kOe})$.
Таблица 1. Значения $H_{\min }(P)$ и погрешности в определении их положений $\Delta H$

\begin{tabular}{l|c|c|c|c|c}
\hline$P(\mathrm{MPa})$ & 32.56 & 48.23 & 72.04 & 88.58 & 106.53 \\
\hline$H_{\min }(P)(\mathrm{kOe})$ & 2.12 & 2.8 & 3.92 & 4.585 & 5.22 \\
\hline$\Delta H(\mathrm{kOe})$ & 0.28 & 0.3 & 0.32 & 0.36 & 0.4
\end{tabular}

Таблица 2. Значения $P_{\min }(H)$ и погрешности в определении их положений $\Delta P$

\begin{tabular}{l|c|c|c|c|c|c|c|c}
\hline$H(\mathrm{kOe})$ & 2.01 & 2.53 & 2.99 & 3.5 & 3.99 & 4.5 & 4.99 & 5.52 \\
\hline$P_{\min }(H)(\mathrm{MPa})$ & 35.4 & 44.6 & 51.0 & 59.0 & 69.5 & 76.5 & 88.5 & 106.1 \\
\hline$\Delta P(\mathrm{MPa})$ & 5 & 5 & 6 & 7 & 8 & 10 & 12 & 15
\end{tabular}

Значения $H_{\min }(P)$ и погрешности в определении их положений $\Delta H$ для кривых на рис. 2 представлены в табл. 1. Табл. 2 содержит величины $P_{\min }(H)$ и $\Delta P$ для кривых на рис. 3.

Теоретические значения $H_{\min }(P)$ и $P_{\min }(H)$ для идеального образца соответствуют точкам ориентационных фазовых переходов [3], поэтому они должны принадлежать одной кривой на плоскости $(P, H)$. Значения $H_{\min }(P)$ из табл. 1 и $P_{\min }(H)$ из табл. 2, как следует из рис. 4 , принадлежат разным кривым, хотя они перекрываются в пределах погрешностей $\Delta H$ и $\Delta P$. Это позволяет считать, что наши экспериментальные данные находятся в количественном согласии с теорией Турова и Шаврова [3], что позволяет их использовать для определения параметров исследуемого гематита. Одним из таких параметров является магнитоупругая константа $B_{66}$, связанная с $H_{\min }(P)$ и $P_{\min }(H)$ соотношениями (15 из [4]:

$$
\begin{gathered}
H_{\min }^{2}(P)+H_{\mathrm{D}} H_{\min }(P)-\alpha_{1} P=0, \\
H^{2}+H_{\mathrm{D}} H-\alpha_{2} P_{\min }(H)=0,
\end{gathered}
$$

где: $H_{\mathrm{D}}=17 \mathrm{kOe}-$ поле Дзялошинского для гематита. Связь параметров $\alpha_{1,2}$ с $B_{66}$ определяется формулами (3.22) из [3] и (11) из [4]:

$$
\alpha=2 B_{66} / C_{66} M_{0} H_{E} .
$$

Здесь: $C_{66}-$ модуль упругости в обозначениях [3], $M_{0}$ - намагниченность подрешетки в гематите, $H_{E}-$ обменное поле. Нижний индекс у $\alpha$ соответствует его значениям, которые определяются уравнением (1) для данных из табл. $1\left(\alpha_{1}\right)$ и уравнением (2) для данных из табл. $2\left(\alpha_{2}\right)$. Значения $\alpha_{1}$ и $\alpha_{2}$ приведены в табл. 3 и табл. 4. Данные табл. 3 и 4 определяют средние значения $\bar{\alpha}_{1}$ и $\bar{\alpha}_{2}$ :

$$
\bar{\alpha}_{1}=1.15 \pm 0.13 ; \quad \bar{\alpha}_{2}=1.18 \pm 0.15,
$$

которые совпадают в пределах меньшей погрешности $\Delta \alpha_{1}=0.13$. Малую разность $\left(\bar{\alpha}_{2}-\bar{\alpha}_{1}\right)$ $=0.03<\Delta \alpha_{1}=0.13$ величин $\bar{\alpha}_{1}$ и $\bar{\alpha}_{2}$, полученных 


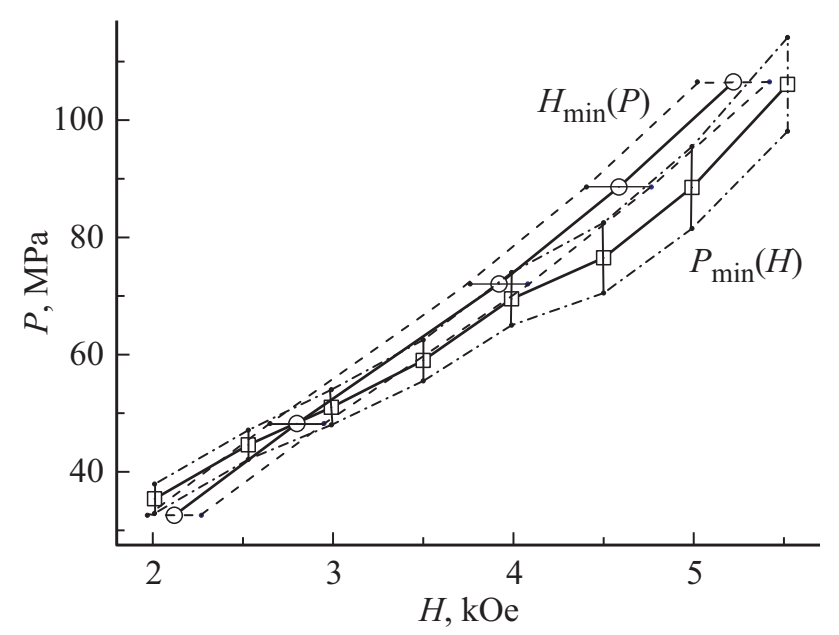

Рис. 4. Зависимости магнитных полей в минимумах скорости квазизвука $\left(H_{\min } V\right)$ от соответствующих величин направленного сжатия $\left(P_{\min } V\right)$. (Область значений, ограниченная штриховой линией, относится к зависимости $V_{H}(P)$, а ограниченная штрихпунктирной — к зависимости $V_{P}(H)$.

разными методами, можно считать подтверждением теории Турова и Шаврова [3].

Однако заметное расхождение кривых $H_{\min }(P)$ и $P_{\min }(H)$ указывает на систематическую погрешность в определении величин $H_{\min }(P)$ и $P_{\min }(H)$, которая в данной теории еще не обсуждалась. Происхождение этой погрешности связано с особенностями ориентационных фазовых переходов при $H=H_{\min }(P)$ и $P=P_{\min }(H)$. Поля $H>H_{\min }(P)$ и давления $P<P_{\min }(H)$ соответствуют высокополевой фазе гематита. Такие поля подавляют действие магнитоупругого поля $H_{P}$ [3], обусловленного давлением, поэтому нескомпенсированный магнитный момент антиферромагнитных подрешеток М ориентирован вдоль $\mathbf{H}(\mathbf{M} \| \mathbf{H})$ (рис. 7 в [3]). Низкополевая фаза гематита реализуется в полях $H<H_{\min }(P)$ и давлениях $P>P_{\min }(H)$. Поле $H_{P}$ в этой фазе способно откло-

Таблица 3. Значения параметра $\alpha_{1}$ и погрешности его определения от величины одноосного давления $P$

\begin{tabular}{l|c|c|c|c|c}
\hline$P(\mathrm{MPa})$ & 32.56 & 48.23 & 72,04 & 88.58 & 106.53 \\
\hline$\alpha_{1}\left(\mathrm{kOe}^{2} / \mathrm{MPa}\right)$ & 1.24 & 1.15 & 1.14 & 1.12 & 1.09 \\
\hline$\Delta \alpha_{1}\left(\mathrm{kOe}^{2} / \mathrm{MPa}\right)$ & 0.18 & 0.14 & 0.11 & 0.11 & 0.1
\end{tabular}

Таблица 4. Значения параметра $\alpha_{2}$ и погрешности его определения от величины магнитного поля $H$

\begin{tabular}{l|l|l|l|l|l|l|l|l}
\hline$H(\mathrm{kOe})$ & 2.01 & 2.53 & 2.99 & 3.5 & 3.99 & 4.5 & 4.99 & 5.52 \\
\hline$\alpha_{2}\left(\mathrm{kOe}^{2} / \mathrm{MPa}\right)$ & 1.08 & 1.11 & 1.17 & 1.22 & 1.21 & 1.26 & 1.24 & 1.17 \\
\hline$\Delta \alpha_{1}\left(\mathrm{kOe}^{2} / \mathrm{MPa}\right)$ & 0.15 & 0.12 & 0.14 & 0.14 & 0.14 & 0.17 & 0.17 & 0.17
\end{tabular}

нить М от направления Н на конечный угол $\varphi$ (рис. 7 в [3]), который можно выбрать в качестве параметра порядка для обсуждаемого фазового перехода. Из сказанного следует, что скорости $V_{P}(H)$ (рис. 2) и $V_{H}(P)$ (рис. 3) относятся к качественно различным кривым. Первые с ростом $H$ описывают переходы из низкополевой фазы в высокополевую, а вторые с ростом $P$ описывают обратный переход. Значения этих скоростей совпадают в точках ориентационного фазового перехода для однородных веществ. Неоднородности, существующие в реальных веществах, превращают эти точки в области фазовых переходов. Зависимости $V_{P}(H)$ и $V_{H}(P)$ достигают минимальных значений в определенных точках этой области, которые не обязательно совпадают. Их несовпадение - одна из причин систематической погрешности, обусловленной различием кривых $H_{\min }(P)$ и $P_{\min }(H)$ на рис. 4. Несовпадение заметно возрастает, когда величина направленного сжатия достигает значения $P \sim 70 \mathrm{MPa}$. Более выражено это на зависимости $P_{\min }(H)$, что определенно свидетельствует в пользу появления дополнительного механизма, обуславливающего это несовпадение. Можно было бы предпринять попытки обобщения теории Турова и Шаврова с учетом неоднородностей вещества в надежде, что это позволит исключить систематическую погрешность и повысить точность магнитоакустических методов. Однако существует еще одна причина несовпадения кривых $H_{\min }(P)$ и $P_{\min }(H)$. Она связана с пластической деформацией, возникающей при использовании больших давлений $P$. Пластическая деформация создает дополнительные неоднородности в веществе, поэтому свойства исследуемого образца оказываются различными для каждой из кривых на рис. 2 и 3. Это значительно затрудняет возможность получения воспроизводимых экспериментальных результатов.

Влияние пластической деформации можно исключить, если ограничиться давлениями $P$, создающими только упругие деформации. По нашим данным (рис. 4) следует ограничиться давлениями $P<70 \mathrm{MPa}$.

\section{4. Выводы}

В настоящей работе приведены результаты измерений магнитоупругого параметра $\alpha$ двумя различными методами: по измерению скорости квазизвука $V_{P}(H)$ (рис. 2) и $V_{H}(P)$ (рис. 3). По результатам этих измерений сделаны следующие выводы:

1. Совпадение результатов, полученных этими методами, в пределах измеряемых и вычисленных погрешностей мы рассматриваем как количественное подтверждение теории Турова и Шаврова [3].

2. Приемлемой точности измерений удалось достигнуть, обеспечив возбуждение только одной ветви магнитоупругих волн за счет тщательной настройки всего акустического тракта. 
3. При оценке величины погрешности определяемых величин учтено уширение точки ориентационного фазового перехода, обусловленного неоднородностями исследуемого образца.

4. Для исключения влияния пластической деформации на воспроизводимость получаемых результатов следует ограничиться давлениями $P<70 \mathrm{MPa}$.

\section{Финансирование работы}

Работа выполнялась в рамках государственного задания Казанского физико-технического института им. Е.К. Завойского - обособленное структурное подразделение Федерального государственного бюджетного учреждения науки „Федеральный исследовательский центр „Казанский научный центр Российской академии наук“ и темы № 01201463332 Федерального государственного бюджетного учреждения науки институт физики металлов им. М.Н. Михеева УрО РАН.

\section{Конфликт интересов}

Авторы заявляют, что у них нет конфликта интересов.

\section{Список литературы}

[1] С.В. Вонсовский. Магнетизм. Наука, М. (1971). 1032 с.

[2] Е.А. Туров, А.В. Колчанов, В.В. Меньшенин, И.Ф. Мирсаев, В.В. Николаев. Симметрия и физические свойства антиферромагнетиков. Физматлит, М. (2001). 560 с.

[3] Е.А. Туров, В.Г. Шавров. УФН 140, 429 (1983).

[4] С.А. Мигачев, Ч.Г. Богданова, М.И. Куркин. ФТТ 57, 37 (2015).

[5] В.И. Ожогин, В.Л. Преображенский. УФН 155, 553 (1988).

[6] Е.А. Туров. ЖЭТФ 96, 2140 (1989).

[7] С.А. Мигачев, М.Ф. Садыков, М.М. Шакирзянов. Письма в ЖЭТФ 91, 372 (2010).

[8] Физическая акустика. / Под ред. У. Мэзона. Мир, М. (1969) T. 6A. 436 c.

[9] П.П. Максименков, В.И. Ожогин. ЖЭТФ 65, 657 (1973).

Редактор Д.В. Жуманов 